\title{
BMJ Open Remote supervision of medical training via videoconference in northern Australia: a qualitative study of the perspectives of supervisors and trainees
}

\author{
Miriam Cameron, ${ }^{1}$ Robin Ray, ${ }^{1}$ Sabe Sabesan ${ }^{1,2}$
}

To cite: Cameron M, Ray R, Sabesan S. Remote supervision of medical training via videoconference in northern Australia: a qualitative study of the perspectives of supervisors and trainees. BMJ Open 2015:5: 006444. doi:10.1136/bmjopen-2014006444

- Prepublication history for this paper is available online. To view these files please visit the journal online (http://dx.doi.org/10.1136/ bmjopen-2014-006444).

Received 25 August 2014 Revised 8 February 2015 Accepted 10 February 2015

\section{(1) CrossMark}

${ }^{1}$ College of Medicine and Dentistry, James Cook University, Townsville, Queensland, Australia ${ }^{2}$ Townsville Hospital, Townsville, Queensland, Australia

\section{Correspondence to}

Associate Professor Sabe Sabesan;

sabe.sabesan@health.qld. gov.au

\section{ABSTRACT}

Objectives: Telemedicine has revolutionised the ability to provide care to patients, relieve professional isolation and provide guidance and supervision to junior medical officers in rural areas. This study evaluated the Townsville teleoncology supervision model for the training of junior medical officers in rural areas of North Queensland, Australia. Specifically, the perspectives of junior and senior medical officers were explored to identify recommendations for future implementation.

Design: A qualitative approach incorporating observation and semistructured interviews was used to collect data. Interviews were uploaded into NVivo 10 data management software. Template analysis enabled themes to be tested and developed through consensus between researchers.

Setting: One tertiary level and four secondary level healthcare centres in rural and regional Queensland, Australia.

Participants: 10 junior medical officers (Interns, Registrars) and 10 senior medical officers (Senior Medical Officers, Consultants) who participated in the Townsville teleoncology model of remote supervision via videoconference (TTMRS) were included in the study.

Primary and Secondary outcome measures:

Perspectives on the telemedicine experience, technology, engagement, professional support, satisfaction and limitations were examined. Perspectives on topics raised by participants were also examined as the interviews progressed.

Results: Four major themes with several subthemes emerged from the data: learning environment, beginning the learning relationship, stimulus for learning and practicalities of remote supervision via videoconference. While some themes were consistent with the current literature, new themes like increased professional edge, recognising non-verbal cues and physical examination challenges were identified.

Conclusions: Remote supervision via videoconference provides readily available guidance to trainees supporting their delivery of appropriate care to patients. However, resources required for upskilling, training in the use of supervision via videoconference, administration issues and nursing support, as well as physical barriers to examinations, must be addressed to enable more efficient implementation.

\section{Strengths and limitations of this study}

- Provisions of patient care, clinical supervision, training and education via videoconference to rural areas have been found to be useful in attracting and retaining health staff. With increased junior medical officers in the workforce, training and supervision of junior medical officers in rural areas are important for safe practice.

- This study provides valuable information regarding user perspectives and organisational implications for supervision of junior medical officers in rural areas via videoconference, which is currently missing from the literature.

- No other studies have used in-depth qualitative methods to describe strengths and limitations of remote supervision via videoconference to rural medical officers, providing important information to support the future implementation of this model.

- While this is a small sample size, the achievement of data saturation indicates that the sample was adequate to address the research question in this context. However, the changes in perspectives over time that could be expected with this new use of videoconferencing technology were beyond the scope of this study.

\section{INTRODUCTION}

Workforce shortages, retention of medical staff, access to adequate supervision and quality healthcare for patients are ongoing concerns in rural Australia. Rural Australians have access to half the number of medical officers per person compared to people in capital cities and medical officers in regional hospitals work on average $6 \mathrm{~h}$ longer per week than their city colleagues. ${ }^{1}$ Studies report that these practitioners often feel isolated while experiencing greater responsibility than their urban counterparts. ${ }^{2-4}$ Moreover, many rural medical officers state that they are forced to make decisions 
without the supervision of senior clinicians, placing additional stress on themselves, while possibly compromising patient care. ${ }^{5-7}$ A study of junior medical officers in rural practice identified that lack of supervision and on-site support, inadequate orientation, limited access to relevant education and the influence of isolation resulted in reduced feelings of professional and personal preparation for rural practice. $^{7}$

Clinical supervision, training and professional support have been thought to be necessary to reduce the feeling of isolation and to attract and retain medical staff in rural areas. ${ }^{8} 9$ In addition, increases in medical student numbers offer opportunities to create training positions in rural areas, although the lack of specialist supervisors in rural areas may be a limiting factor ${ }^{10}$ and concerns have been raised about the variability, quality and time commitment required to provide specialist services to areas that are often difficult to access. ${ }^{11} 12$ Telemedicine models can be used to provide access to professional support for rural doctors and supervision for trainees in rural locations. ${ }^{13} 14$

Telemedicine is defined as the use of information and communications technology to provide healthcare services to individuals who are some distance from the healthcare provider' (ref. 15, p.765). The use of telemedicine has been supported in Australia by many specialist colleges such as the Royal Australasian College of Physicians (RACP) and the Australian College of Rural and Remote Medicine (ACRRM), with over 700 medical officers affiliated with the telemedicine database. Through this media, medical officers and patients in rural areas are provided with access to specialist services via live synchronous based videoconferencing facilities, enabling communication between rural primary healthcare centres and regional or urban tertiary hospitals.

Numerous studies describe successful treatment outcomes and patient satisfaction with medical, nursing and allied healthcare via videoconferencing, including a reduction in expenditure, increased access and quality of care for rural patients. ${ }^{16-23}$ Increased access to education, professional development and networking has also been described. ${ }^{24} 25$ However, very few studies have investigated the supervision of medical officers via videoconferencing. ${ }^{26}{ }^{27}$ Given that supervision of junior medical officers via videoconferencing is occurring in many parts of rural Australia, little is known about junior medical officers' experiences of this style of supervision, or whether they consider remote supervision to be a feasible model for professional development, decision-making and increasing confidence.

The Townsville Cancer Centre (TCC) in North Queensland, Australia, has been providing oncology services via videoconferencing for the past 6 years. This service brings oncology closer to home for rural patients from an area covering over 300000 square kilometres. ${ }^{17}$ As part of this service, rural based medical officers were required to provide medical input to the telemedicine consultation conducted by tertiary specialists, allowing opportunity for videoconference-based interaction, discussion and supervision in many aspects of medical and professional care, as detailed below. In a TCC study of medical oncology clinics via videoconferencing, medical officers welcomed this model for educational and professional benefits. Based on the observed educational benefits for the doctors, in the past 2 years, a remote supervision model was established and incorporated into the service delivery model. ${ }^{28}$

\section{TOWNSVILLE TELEONCOLOGY MODEL FOR REMOTE SUPERVISION (TTMRS)}

In this model, junior medical officers (interns and medical registrars) in rural areas are provided with one-on-one supervision by senior medical officers in regional tertiary centres via videoconference prior to, during and after consultations. The act of supervision here is defined as the process of observing, teaching, discussion and direction about how patients are managed. As part of the training programme, an orientation to remote supervision via videoconference, teleoncology services and a handbook for junior medical staff are provided for all junior medical officers. Currently, there is no prior face-to-face contact between oncologists and trainee junior medical officers.

Every case incorporates a case based discussion, enabling remote supervision to include a range of services such as specialist clinics on demand, patient reviews and inpatient admissions. Training in medical management of patients with complex oncology diagnoses and a variety of medical topics relevant to junior doctors are covered weekly. Therefore, patient consultations, medical supervision and training are all covered within this model. This is a unique opportunity for rural medical officers and is unlike the face-to-face model in that varying levels of specialist patient care are discussed and taught to rural-based medical staff.

At rural sites that have trainees, junior medical officers also have access to supervision by local senior medical officers (rural generalists and general practitioners) on site to corroborate findings, provide ongoing support and guidance and discuss patient management. While the senior medical officers in the rural sites do not have specialist knowledge in oncology, they are able to attend to trainees' concerns regarding management of nononcology issues when required. This model of remote supervision via videoconference, in combination with local senior medical officers, fulfills its requirements for supervision and training set by several medical colleges in Australia, including the basic training programme of the RACP and the ACRRM, as well as internship accreditation bodies.

\section{AIM}

This study evaluated the Townsville teleoncology model for remote supervision (TTMRS) for the training of junior medical officers in rural areas. Specifically, we 
explored the perspectives of junior medical officers in rural areas and their supervisors who utilised remote supervision via videoconferencing.

Subquestions included:

- How do junior medical officers perceive remote supervision via videoconference?

- How do senior medical officers perceive remote supervision via videoconference?

- What recommendations can be made to ensure quality supervision via videoconferencing?

\section{METHOD}

A descriptive qualitative approach was undertaken using observations, semistructured interviews and template analysis in a thematic analysis framework. The template, devised from the literature and professional practice, provided an organised approach to theme development and testing based on the topics explored in the interviews. Templates are created to generate and test themes in a logical manner, enabling major themes and subthemes to be generated. ${ }^{29-31}$ While one of the researchers (SS) is integral to the supervisory model being explored in this project, neither of the other two researchers (MC and RR) has any professional ties to the programme. Any bias in the template that may have resulted from researcher three's involvement was mitigated by researcher one (MC) undertaking the initial analysis and the team approach to verification of theme generation. Also, when new topics arose in early interviews, they were verified in successive interviews, building a wider range of topics and expanding potential outcomes.

\section{SAMPLE/PARTICIPANTS}

Junior medical officers in four different rural sites (interns and internal medicine registrars), senior medical officers (rural generalists and general practitioners) in rural sites and oncologists at TCC who had participated in the TTMRS were invited to participate in the study. These four rural sites enabled data to be drawn from locations where junior medical officers are working. Other sites participating in the TTMRS are only serviced by senior medical officers and thus were not relevant for the purposes of this study. Rural junior and senior medical officers were purposely sampled through an email invitation. Twenty (10 junior and 10 senior) medical officers indicated their willingness to participate in the study, which was the entire sample frame. Participants were sent information and consent forms prior to the interview and consent was confirmed at the time of interview. All agreed to being recorded with a digital voice recorder (Olympus VN-712PC).

\section{DATA COLLECTION}

Observations and field notes were recorded by MC during two teleoncology supervision sessions in 2013. These data were analysed and applied to the construction of a semistructured interview guide that was pilot tested with four medical officers and questions refined based on their feedback. Semistructured interviews enabled us to capture important information while providing flexibility to investigate unexplored topics in this new area of research. As the interviews progressed, analysis of data contributed to additions to the interview guide enabling us to verify emerging themes. ${ }^{30}{ }^{32}$ The interview guide explored experience with telemedicine, technology, engagement, confidence, professional development, support and supervision, improvements, perception of costs and challenges. Examples of questions can be found in table 1 .

Twenty semistructured interviews were undertaken during 2013 and 2014, 10 with junior medical officers and 10 with senior medical officers. Four interviews were conducted face to face and 16 via telephone, each lasting between 20 and $35 \mathrm{~min}$. The interviews were transcribed within $72 \mathrm{~h}$, uploaded into QSR NVivo 10 data management software and coded. ${ }^{33}$

\section{DATA ANALYSIS}

Transcribed interviews were coded collaboratively by MC, RR and SS, with broad themes identified as

\begin{tabular}{ll} 
Table 1 Semistructured interview schedule \\
\hline Topic areas & Question examples \\
\hline Telemedicine experience & $\begin{array}{l}\text { Can you tell me about your experiences using telemedicine and remote supervision via } \\
\text { videoconference? }\end{array}$ \\
$\begin{array}{ll}\text { Technology } & \text { How did you find the visual and audio quality during the supervision and training sessions? } \\
\text { Engagement/rapport/ } & \text { Were you able to build rapport or engage with the physician on the other end? What experience } \\
\text { confidence } & \text { do you have with this compared to face to face? }\end{array}$ \\
$\begin{array}{ll}\text { Professional support/ } & \text { How do you feel the remote supervision experience has shaped your professional development? } \\
\text { guidance } & \text { How do you feel remote supervision contributes to the health budget? } \\
\text { Perception of costs } & \text { Can you describe your overall feelings towards remote supervision via videoconference for rural } \\
\text { Overall satisfaction } & \text { medical officers? How do you see it working in the future? }\end{array}$ \\
$\begin{array}{l}\text { Limitations/challenges } \\
\text { Ddditional }\end{array}$ & $\begin{array}{l}\text { Do you feel there are any limitations or challenges with this type of supervision? } \\
\text { Is there anything else you would like to comment on? }\end{array}$
\end{tabular}


interviews progressed. This allowed emerging topics to be explored in subsequent interviews. No new themes emerged in the final five interviews, indicating data saturation. During initial analysis, large groups of codes were generated (20). All researchers identified possible exceptions and redundancies in the initial coding and worked to arrange the codes into more theoretically dense groupings. Themes were identified and discrepancies discussed by the research team to reach consensus. Finally, the relationships between the themes and codes were explored using mapping and flow charts. This process highlighted relationships and associations between different concepts.

\section{QUALITY CONTROL}

Many researchers within the qualitative field place emphasis on the trustworthiness of qualitative findings and the internal and external factors which can contribute to them. ${ }^{34}{ }^{35}$ This study used a number of safeguards. Two research team members (MC and RR) analysed a sample of the data to identify quotes and pertaining codes which underpinned major themes. Further consensus by all team members was achieved in relation to major and subthemes. This triangulation approach for inter-rater reliability ensured interpretative rigour. ${ }^{35}{ }^{36}$ As researcher one (MC) had interviewed all participants, the analyses of interviews allowed for the use of multiple data sources (direct observation, transcripts and recall), which is considered a key factor in ensuring trustworthiness. ${ }^{36}$

\section{RESULTS}

The demographic characteristics of all 20 junior and senior medical officers are summarised in table 2.

Data analysis revealed four major themes relating to remote supervision via videoconference. Within each theme, several subthemes emerged, revealing positive and negative aspects. Reciprocal comments by junior and senior medical officers were noted among each theme, with minor differences noted within two major themes (isolation and physical examinations) between rural and Townsville based senior medical officers and oncology consultants. Major themes and subthemes with examples can be viewed in table 3 .

\section{Learning environment}

All 20 participants expressed positive attitudes towards remote supervision via videoconferencing and the need to continue this form of supervision in the future. Participants felt that this supervision model could be expanded to other areas of medicine such as internal medicine, psychiatry and cardiology.

\section{Personal attributes and communication style}

Eight junior medical officers and seven senior medical officers identified that personal attributes and communication style contributed to a lasting positive interaction for remote supervision. Attributes such as enthusiasm,

\begin{tabular}{|c|c|c|}
\hline Characteristics & $\begin{array}{l}\text { (Senior } \\
\mathrm{n}=10)\end{array}$ & $\begin{array}{l}\text { (Junior } \\
\mathrm{n}=10 \text { ) }\end{array}$ \\
\hline \multicolumn{3}{|l|}{ Gender } \\
\hline Male & 7 & 5 \\
\hline Female & 3 & 5 \\
\hline \multicolumn{3}{|l|}{ Age } \\
\hline $20-25$ & - & 2 \\
\hline $26-30$ & - & 3 \\
\hline $31-35$ & - & 4 \\
\hline $36-40$ & 3 & 1 \\
\hline $41-45$ & 6 & - \\
\hline $46-50$ & 1 & - \\
\hline \multicolumn{3}{|l|}{ Position status } \\
\hline Intern & - & 3 \\
\hline Registrar in training & - & 7 \\
\hline Rural based senior medical officer & 5 & - \\
\hline Oncologist (TCC) & 5 & - \\
\hline \multicolumn{3}{|l|}{ Years of medical experience } \\
\hline $0-2$ & - & 3 \\
\hline $3-5$ & - & 6 \\
\hline $6-8$ & - & 1 \\
\hline $9-11$ & 5 & - \\
\hline $12-14$ & 1 & - \\
\hline $15+$ & 4 & - \\
\hline \multicolumn{3}{|l|}{$\begin{array}{l}\text { Years of teleoncology/ } \\
\text { videoconference experience }\end{array}$} \\
\hline $0-1$ & - & 6 \\
\hline $1-2$ & - & 3 \\
\hline $2-3$ & - & 1 \\
\hline $3-4$ & - & - \\
\hline $4-5$ & 4 & - \\
\hline $5+$ & 6 & - \\
\hline
\end{tabular}

encouragement, motivational approach and friendliness were often mentioned. They also described the need for clear and open communication, active listening and attentiveness to ensure positive and fulfilling interactions, with increased emphasis when using remote supervision via videoconference.

\section{Relationships}

Eighteen medical officers (9 junior physician JDs, 9 senior physician SDs) considered that supervision via videoconference was an important way to provide stronger relationships and develop good rapport between rural junior medical officers and their supervisors. They described the importance of visualising the other physician on a weekly basis and the significance of this continued support.

\section{Practice validation}

All 10 junior medical officers identified that supervision via videoconference validated their practice and management of patients, giving them increased confidence 


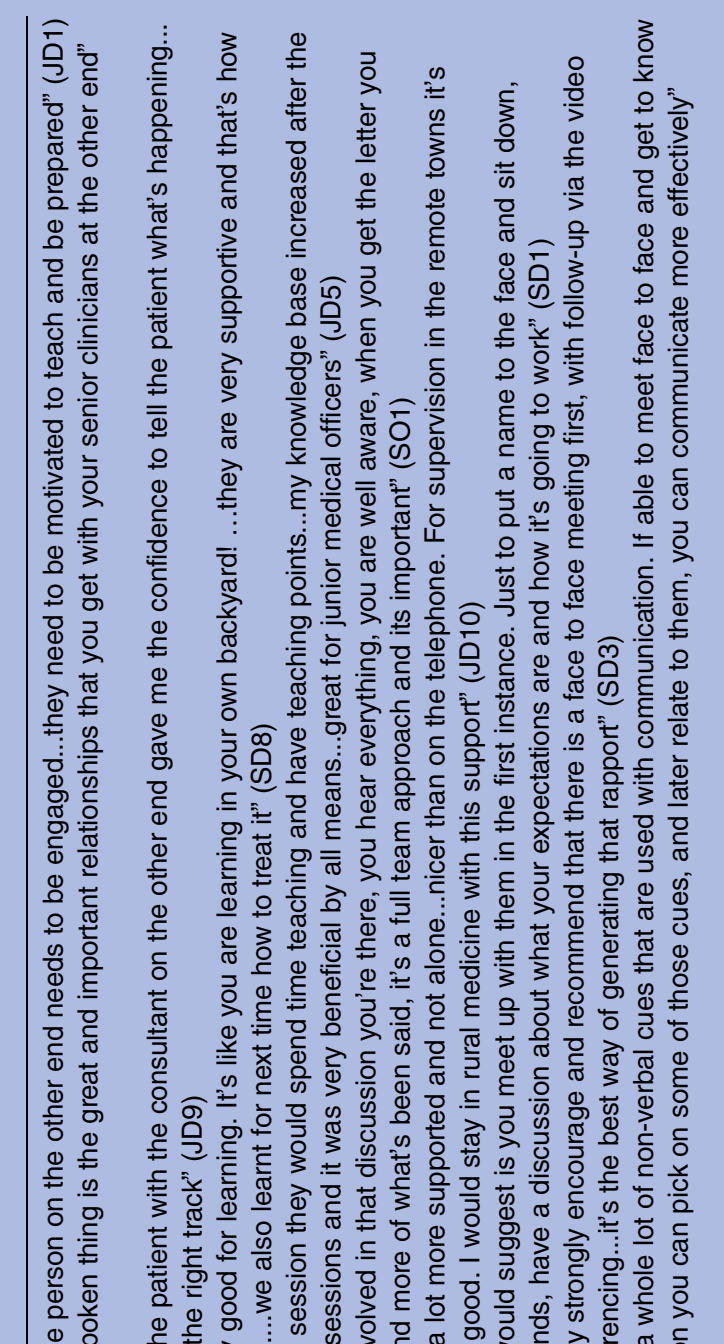

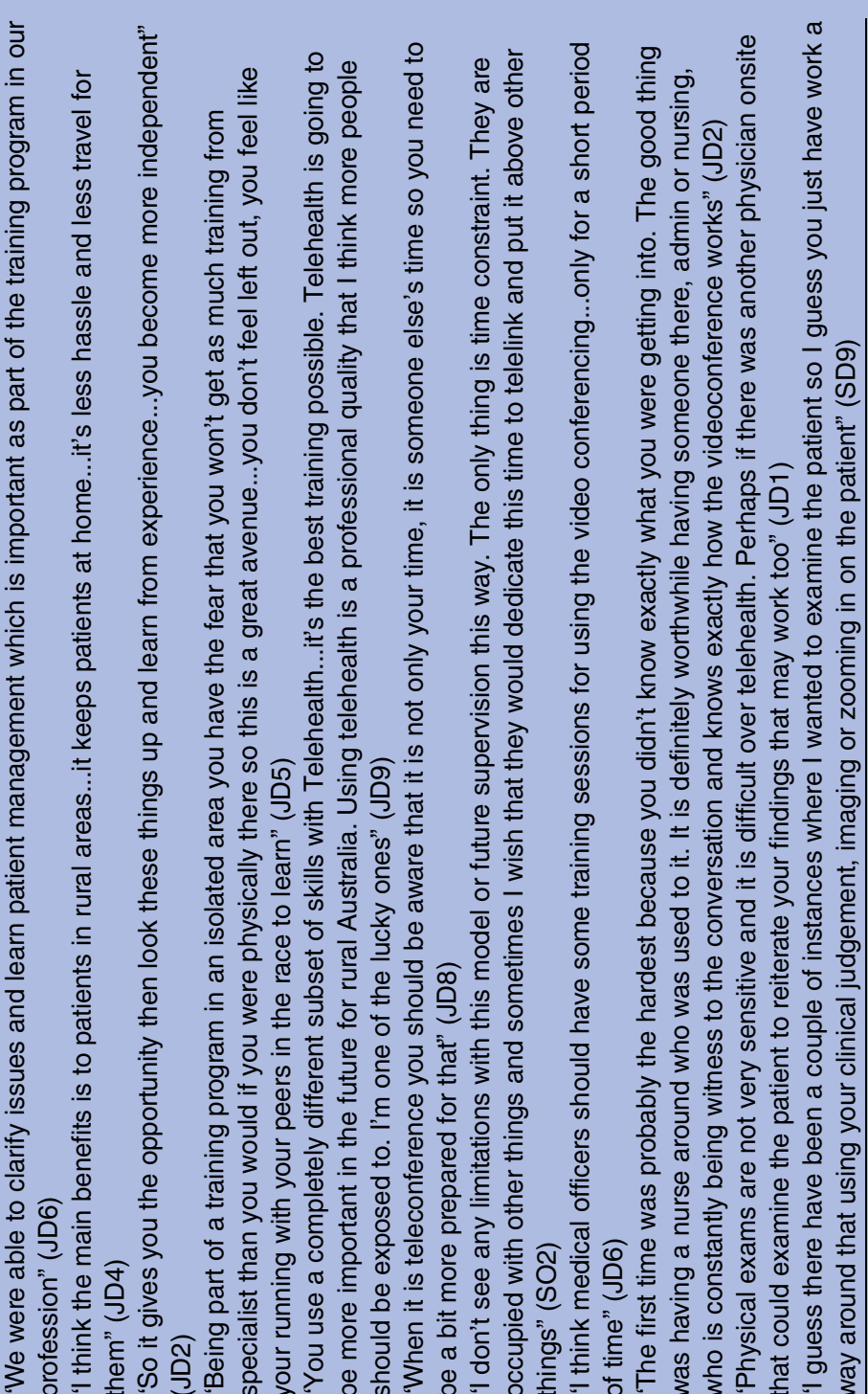

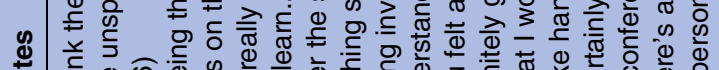

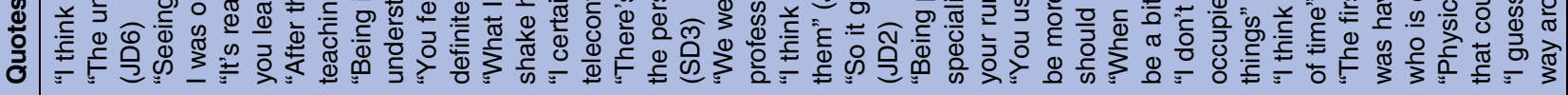
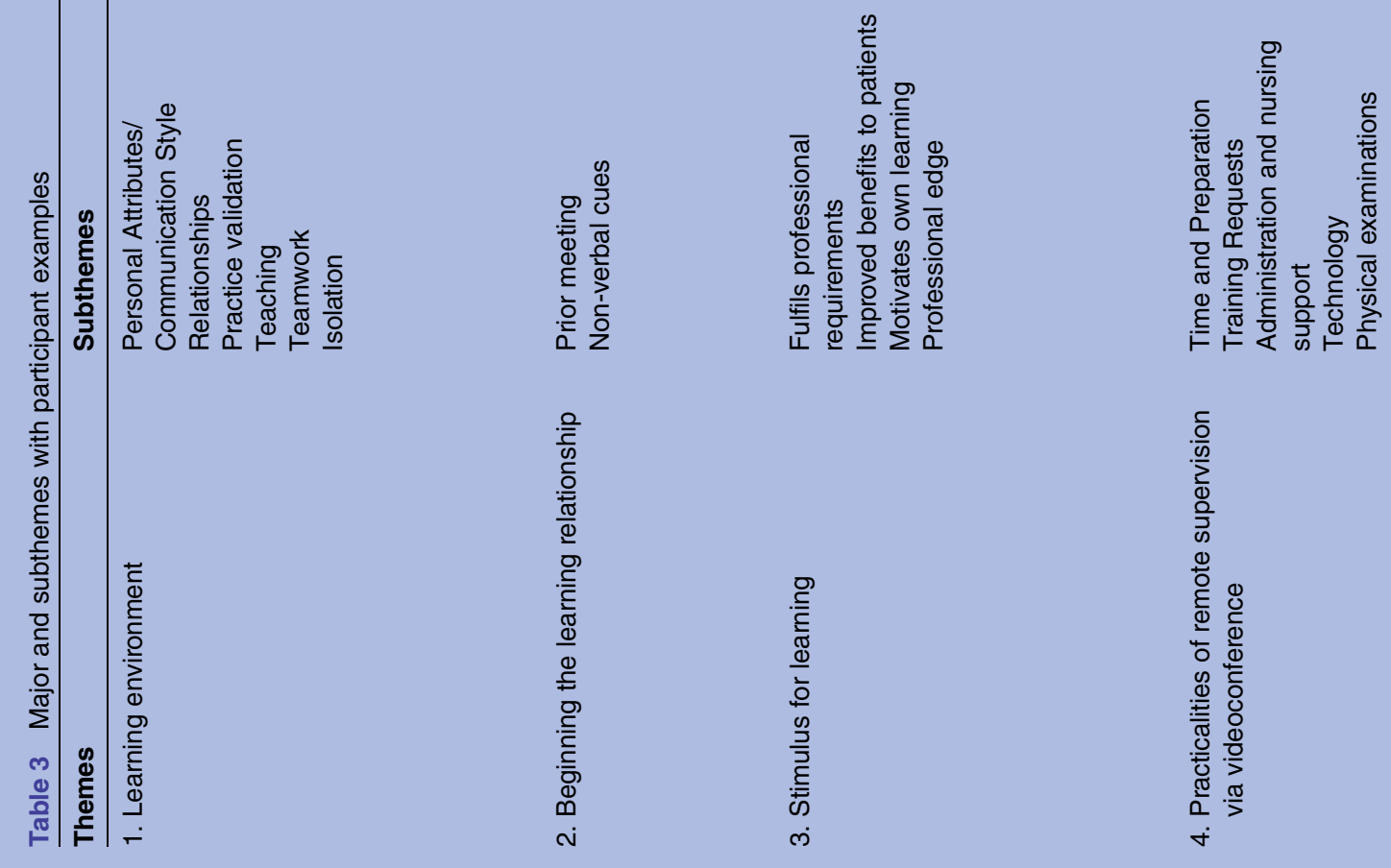
in future medical management. This feedback helped them to feel supported in their decision-making.

\section{Teaching}

Eight junior medical officers identified positive aspects of teaching following patient review, teaching and consultation sessions. These additional teaching sessions increased their knowledge base and confidence with patient management. During teaching sessions, they were able to clarify issues or gain a broader understanding of subspecialty fields of medicine. The importance of effective teaching was also highlighted by senior medical officers in that it enhanced their professional development, and contributed to building a positive learning environment. All medical officers were able to learn about different aspects of clinical cases while developing an ongoing clinical relationship.

\section{Teamwork}

Being part of a team and witnessing the overall care of a patient was a consistent subtheme across the data. Five senior medical officers explained that multidisciplinary telemedicine involved them in a team approach in which they could observe several aspects of patient care, including allied health, not usually available with a traditional face-to-face supervisory model. Junior medical officers concurred with this notion, expressing that being exposed to many areas of patient care, medical and allied health, contributed to a broader understanding of the multifaceted nature of patient care.

\section{Isolation}

Twelve medical officers (8 JDs, 4 SDs-rurally based) noted a reduction in feelings of isolation induced by the availability of senior support through videoconference. Reasons for this included increased access to help, improved confidence and establishment of trustworthy relationships between medical officers. Rural senior medical officers described the importance of being able to collaborate with seniors in tertiary centres, while providing supervision to the junior medical officers, highlighted through their responses.

\section{Beginning the learning relationship}

Many important requirements for beginning the learning relationship between and supervisor and junior physician were identified across the data.

\section{Prior meeting and nonverbal cues}

Fourteen medical officers ( 7 JDs, 7 SDs) indicated their preference for a face-to-face meeting, prior to the establishment of videoconference-based supervision sessions, to develop rapport and learn about a person's nonverbal cues. Senior medical officers (5) suggested that a prior meeting would help understand a person's non-verbal cues as these are often difficult to see over videoconference and this information would assist in developing a deeper understanding of the person.
The remaining six junior and senior medical officers did not feel strongly either way about the usefulness of a prior meeting.

\section{Stimulus for learning}

Motivational aspects that encourage participation and learning via videoconference were explored through participant responses.

\section{Fulfils professional requirements}

Participating in a supervision session via videoconference helped to fulfil professional accreditation and provide ongoing quality support and care for patients in rural areas. Junior medical officers noted that they were able to gain access to specialist advice and improve their own technological skills, equipping them for future work.

\section{Improved benefits to patients}

All twenty medical officers mentioned benefits to patients in various ways. Junior medical officers described how the provision of specialist advice and teaching enabled better provision of care to patients in rural areas, as it increased their confidence and autonomy for patient intervention. Senior medical officers concurred with comments regarding improvements to overall patient care and management by the junior staff. They suggested that this ability to provide direct advice to junior staff, in real time, ensured that patient care was similar to levels delivered in tertiary centres.

\section{Motivates self-directed learning}

Seven junior and six senior medical officers agreed that set supervision and teaching sessions with medical officers in rural areas motivated their own autonomous learning. Juniors were inspired to read additional material and improve their evidence-based practice following discussions with seniors.

\section{Professional edge}

Most junior medical officers raised the professional advantage gained from remote supervision via videoconference. All medical trainee registrars suggested that the teleoncology supervision model gave them a professional advantage compared with their trainee peers, as they were provided with knowledge and training within a specialist area. Although some were initially concerned that being in a rural centre would disadvantage their learning, they noted that having the ability to witness multidisciplinary care and specialist intervention improved their knowledge. In addition, having the technological ability to provide care to rural sites would equip them for the future as they recognised that technology would be the mainstay of providing consultations. "Having the support from the specialist has been really good, you don't necessarily get that opportunity to have one to one time with a specialist consultant in the big hospitals...its given me confidence...It's given me the edge" (JD7). 
Practicalities of remote supervision via videoconference

Both positive aspects and challenges in the provision of supervision via videoconference were evident in all interviews. Comments and suggestions for future implementation were made with common subthemes arising from the data.

\section{Time and preparation}

Common issues raised by all participants were time commitment and preparation. All junior medical officers and seven senior medical officers said that there was increased time commitment within videoconferencebased supervision compared with face-to-face supervision. This included increased waiting times caused by technological difficulties or supervisor availability. Juniors stated that in rural sites, there is an additional time pressure, especially if there are few medical officers caring for numerous patients. Participants suggested that a consistent time with defined goals for the session would meet requirements more efficiently. Seniors supported this approach with the expectation that junior medical officers prepare in advance to increase time efficiency of sessions.

\section{Training requests}

The desire for training in use of the technology was expressed by 18 medical officers ( 8 JDs, 10 SDs). The majority identified the necessity of a short training session on technical aspects such as camera and audio equipment. Increased teaching in specialty fields and general medicine was requested by nine junior medical officers to expand professional support and guidance required to maximise the effectiveness of supervision via videoconference.

\section{Administration and nursing support}

Sixteen medical officers ( 8 JDs, 8 SDs) cited the need for increased administration support from rural and regional sites to improve efficiency. Nursing support was also highly sought after by the majority of junior medical officers, as nurses were consistently able to provide prior knowledge of the patients. Therefore, having a consistent person to assist and advise in sessions would provide better patient care and improve multidisciplinary team learning for the junior medical officers. However, this is resource intensive in a context that often operates on minimal staffing.

\section{Technology}

Overall, the video and audio quality of videoconferencing in the teleoncology model was rated highly by all medical officers, with only occasional minor discrepancies mentioned by two junior medical officers, which included disruptions in connections between sites. Having a high quality system, as is available across most sites in regional northern Australia, was extremely important for providing appropriate supervision and care to patients. In addition, the ability to show radiological images and utilise zoom technology to view patient attributes was essential to validate physical examinations and facilitate learning.

\section{Physical examinations}

The inability of a senior supervisor to physically examine a patient was a challenge identified by nine junior medical officers. This was one of the main limitations of remote supervision via videoconference compared with a face-to-face model. In oncology, the physical exam was stated as being less important as imaging has become more reliable in determining patient status. However, four rurally based senior medical officers who worked with junior medical officers suggested that one solution could be asking another physician in the rural site to verify the examination findings, or to use the zoom capability of the videoconferencing technology to help view patients and physical signs.

\section{DISCUSSION}

This study explored the perspectives of junior and senior medical officers in rural areas regarding remote supervision via videoconference. All medical officers expressed positive attitudes and conveyed suggestions for the future implementation of models of remote supervision via videoconference for trainees across teleoncology and other areas of medicine. While some themes arising from this study are similar to those reported previously, this study identified additional themes relevant to the provision of videoconference-based supervision. Two minor themes (isolation and physical examinations) showed differences in perceptions between senior medical officers based in rural sites and Townsville based senior oncologists.

Advantages of remote supervision via videoconference included the ability to build relationships and establish rapport between supervisors and trainees, and improved communication between rural and tertiary facilities. Through this model of supervision, trainee and senior medical officers in rural areas felt decreased isolation, increased their knowledge of patient care, experienced validation of their current and future practice management, and increased their knowledge base about various aspects of medical and allied health patient care. This was noted to fulfil their own professional requirements, while providing a professional advantage over peers who had not experienced this form of supervision.

Disadvantages of remote supervision via videoconference included the requirement for efficient time management skills, increased administration, nursing support, training and preparation prior to videoconferencing sessions. While technological issues were seldom reported, the ability of a centre to provide high-level technology was an important factor for future implementation of a quality service between rural and tertiary centres. Physical examination barriers were noted as a limitation for junior medical officers in rural areas. This 
physicality issue may be overcome by asking senior medical officers in rural areas to concur with findings, or using zoom or imaging technology to help determine patient status.

Several elements were found to increase the usefulness of remote supervision via videoconferencing. Positive personal and communication style are attributes which contributed to the learning environment. These included enthusiasm, encouragement and active listening skills which were identified by the majority of medical officers. Relationships and teamwork are also critical aspects seen as essential to establishing rapport throughout the sessions. Participants indicated that the working relationship was best established with consistent weekly sessions. They appreciated the value of teamwork and involvement in patient care while learning different general and specialist approaches to patient management. Our findings are consistent with other studies, highlighting the need for good communication and positive personal attributes, as well as the importance of positive engagement between supervisors and supervisees, an attribute essential for effective collaboration and cohesion within a videoconference-based supervision model. ${ }^{37-39}$ Our study also contradicts others' work which found poorer relationship and rapport building between medical officers. ${ }^{37} 40{ }^{41}$ However, our model differs in several aspects. The TTMRS is integrated as a part of routine clinical work and educational sessions are done on a regular basis (at least weekly), so that there is enough time to develop a relationship. These previous studies did not have consistent sessions between participants. Therefore, training for supervisors undertaking remote supervision models needs to incorporate consistent sessions that provide encouragement to trainees through the videoconference based system.

Increased opportunity for interaction, expanded learning and teamwork have been identified as advantages of supervision via videoconferencing. ${ }^{40} 42 \quad 43$ Using semistructured interviews, our study explored these aspects in depth, further validating that through remote supervision via videoconferencing, these opportunities exist and are welcomed. Through the TTMRS, rural junior medical officers are exposed to and witness a variety of patient care approaches, not necessarily available in a face-to-face model in a tertiary general medicine unit, thus increasing learning opportunities. Other benefits included reduced feelings of isolation, increased confidence through practice validation and the advantages of providing support and education directly to the junior medical officers' location, negating the need for timeconsuming travel. Our findings concur with other studies into general medicine, supporting the case for education and supervision via videoconferencing, without the need for supervisors being co-located with trainees. ${ }^{25} 4044-46$

The importance of establishing the learning relationship through a prior face-to-face meeting to develop rapport and learn about non-verbal cues may be considered when developing a remote videoconferencebased supervision model. ${ }^{42}$ In our study, some medical officers suggested that a prior face-to-face meeting may enable non-verbal cues to be recognised in subsequent videoconferencing and help to clarify visual and social cues. A previous study had similar findings, with participants indicating a decreased sense of personal communication style through videoconferencing. ${ }^{37}$ However, their study looked at pairs of medical officers who already knew each other prior to the study. Considering that the participants in our study did not know each other prior to supervision sessions, a prior face-to-face meeting may be important for development of relationships in future models of remote supervision via videoconference.

Stimulus for learning was another major theme explored in this study. Remote supervision via videoconference was noted to help fulfil professional requirements while also stimulating motivation for autonomy and self-directed evidence-based learning. Through remote supervision, junior medical officers providing generalist care were able to achieve higher levels of confidence, increase their knowledge base and address specific specialist issues relevant to patient care. This increased knowledge, and confidence assisted their management of patients and validated their ongoing practice. Practice validation through increased clinical support, particularly for rural medical officers, has previously been noted by Sargeant et al. ${ }^{38}$ However, their study did not explore practice validation in great detail. In our study, the use of semistructured questions allowed further exploration of these factors and found that through provision of consistent supervision via videoconference, junior medical officers in rural areas may feel validated and confident in their ongoing management of patients.

Perhaps the most noteworthy new finding from our study is the notion of 'professional edge', a theme which has not been mentioned in previous studies. Junior medical officers, especially trainee registrars, indicated their ability to gain increased knowledge in specialist oncology areas and multidisciplinary support through telehealth models of care as part of their internal medical rotation in rural areas. They described how this provided them with an increased set of skills which they identified as an advantage over their peers, something they would not have developed in an urban internal medicine setting. While some reported that they were initially reluctant to go to a rural area, as it may disadvantage their learning, many concluded that in fact their learning in oncology was increased compared with staying in an internal medicine rotation at a tertiary centre. Promotion of this outcome (professional edge) from our study could have implications for recruitment and retention of medical officers to rural and remote areas. This notion of increasing 'professional edge' for trainees warrants further exploration in future research.

The economic advantage of telemedicine, with reduction in costs to patients, has been reported and 
hypothesised in several studies. ${ }^{16} 4748$ However, there is considerable paucity of literature regarding the costs of medical training and supervision via videoconference. ${ }^{26}$ Studies suggest that group supervision and education to multiple sites would reduce costs, allowing health workers to surpass geographical barriers and increase contact between colleagues. ${ }^{42}$ In our study, participants endorsed the concept that remote supervision saved their own travel time. However, formal economic analysis is required to assess the costs of these types of supervision models. Future cost benefit analysis could use quantitative and qualitative methods incorporating many variables such as additional staff costs, cost of equipment and maintenance and costs associated with staff travel for training.

Several issues need to be addressed to improve the efficiency and usefulness of remote supervision via videoconference. The increased time commitment and preparation required for a supervision session was a challenge for many rural medical officers. However, having a consistent time for supervision with focused goals may be a way to reduce wasted time and improve session outcomes. The provision of training, administration, nursing and technological support is also required to increase efficiency. While technology has been seen as a barrier by some, ${ }^{26}$ the technology used in the TTMRS was largely viewed as positive with advanced audio and video quality. This was seen as important for further learning and would help with aspects of teaching, particularly those associated with physical examinations. ${ }^{37} 49$ Despite these challenges, improving training accessibility for rural medical officers is essential for improving education and patient care, a notion supported by health workers in Indigenous communities, with similar geographic constraints as this study. ${ }^{50}$

\section{Limitations of the study}

A limitation of the study was that most participants were part of an established teleoncology network in North Queensland where the technology was already in place and long-standing relationships had been established between TCC and rural sites. Therefore, implementation of a remote supervision model like this in other centres will require the installation of quality equipment and the training of staff in its use as well as the establishment of working relationships between rural and tertiary sites. Each participant gave one interview; thus, experiences over time could not be captured. A future longitudinal study would be a useful addition to the data set. The influence of past exposure to videoconferencing and number of consultations may also be useful in future studies. This sample was a heterogeneous group of participants. The sample size was small (n20); however, data saturation was achieved, indicating that the sample was adequate for addressing our research question. While one of the researchers (SS) is integral to the development of the supervisory model, any bias that may have resulted was mitigated through researcher one (MC) and researcher two (RR), taking a pivotal role within initial data collection and analysis.

\section{CONCLUSION}

Junior and senior medical officers participating in the TTMRS described many positive aspects of the model and identified issues that need to be addressed. Advantages for remote supervision via videoconference include the provision of increased confidence, new learning opportunities, practice validation, professional support, establishing relationships and decreased feelings of isolation for junior and senior medical officers in rural areas. Disadvantages included technological issues, increased administration and nursing support, increased time commitment and preparation and limitations to physical access by senior medical specialists in rural areas. New information such as the need for prior face-to-face meetings, consistent sessions and professional edge was also found.

Further research into the value of consistent sessions, the professional edge and the cost-effectiveness of this model is required. However, the outcomes of this study provide important considerations for the further development of models of remote supervision across other medical fields, time allocation for teaching following consultation and the ongoing support for junior staff working in rural facilities.

Contributors All authors have made substantial contributions following the criteria outlined by ICMJE. MC contributed to the conception and design of the project, obtaining ethics approval, data collection, analysis, drafting, writing and revision of the manuscript. RR supervised and contributed to conception and design, analysis and interpretation of the data and revisions of the written manuscript. SS supervised and contributed to conception and design, analysis and interpretation of the data and revisions of the written manuscript.

Funding Funding has been provided by the James Cook University Bachelor of Medicine, Bachelor of Surgery (Honours) program (Ref. no: 333181). The researchers are independent of the funders of the research.

Competing interests MC, RR and SS have support from (James Cook University) for the submitted work.

Provenance and peer review Not commissioned; externally peer reviewed.

Data sharing statement Data including qualitative code and the data set can be made available by the corresponding author on request.

Ethics approval Ethical approval for the study was obtained by the Townsville Hospital and Health Service Human Research Ethics Committee (HREC/13/ QTHS/46) and the James Cook University Human Research Ethics Committee (H5407).

Open Access This is an Open Access article distributed in accordance with the Creative Commons Attribution Non Commercial (CC BY-NC 4.0) license, which permits others to distribute, remix, adapt, build upon this work noncommercially, and license their derivative works on different terms, provided the original work is properly cited and the use is non-commercial. See: http:// creativecommons.org/licenses/by-nc/4.0/

\section{REFERENCES}

1. AlHW. Rural, regional and remote health: indicators of health system performance. 2008.

2. Hays RB, Sen Gupta T, Arlett K. Integrating general practice medical education. The North Queensland model. Med J Aust 1994;160:388-9. 
3. Incitti F, Rourke J, Rourke LL, et al. Rural women family physicians. Are they unique? Can Fam Physician n 2003;49:320-7.

4. Francis K. Health and health practice in rural Australia: where are we, where to from here? Online J Rural Nurs Health Care 2005;5:28-36.

5. Idema R, Brownhill S, Haines M, et al. 'Hands on, hands off': a model of clinical supervision that recognises trainees' needs for support and independence. Aust Health Rev 2010;34:286-91.

6. Alison S. Garry D, Carl BC, Retention of rural physicians: tipping the decision-making scales. Can J Rural Med 1998;3:209-16.

7. Smith DM. Barriers facing junior doctors in rural practice. Rural Remote Health 2005;5:348-55.

8. Alexander CF, Fraser JD. Education, training and support needs of Australian trained doctors and international medical graduates in rural Australia: a case of special needs? Rural Remote Health 2007;7:681.

9. Daly M, Perkins D, Kumar K, et al. What factors in rural and remote extended clinical placements may contribute to preparedness for practice from the perspective of students and clinicians? Med Teach 2013;35:900-7.

10. Australia HW. Health workforce 2025-doctors, nurses and midwives-volume 1. 2012

11. Wilson NW, Couper ID, De Vries E, et al. A critical review of interventions to redress the inequitable distribution of healthcare professionals to rural and remote areas. Rural Remote Health 2009;9:1060.

12. Underhill C, Bartel R, Goldstein D, et al. Mapping oncology services in regional and rural Australia. Aust J Rural Health 2009;17:321-1.

13. Hazin R, Qaddoumi I. Teleoncology: current and future applications for improving cancer care globally. Lancet Oncol 2010;11:204-10.

14. Pathmanathan S, Burgher B, Sabesan S. Is intensive chemotherapy safe for rural cancer patients? Intern Med J 2013;43:643-9.

15. Roine R, Ohinmaa A, Hailey D. Assessing telemedicine: a systematic review of the literature. CMAJ 2001:165:765-71.

16. Thaker DA, Monypenny R, Sabesan S. Cost savings from a telemedicine model of care in northern Queensland, Australia. Med J Aust 2013;199:414-17.

17. Sabesan S, Larkins S, Evans R, et al. Telemedicine for rural cancer care in North Queensland: bringing cancer care home. Aust J Rural Health 2012;20:259-64.

18. Blignault I. Multipoint videoconferencing in health: a review of three years' experience in Queensland, Australia. Telemed J 2000;6:269-74.

19. Dillon $\mathrm{E}$, Loermans J, Davis $\mathrm{D}$, et al. Evaluation of the Western Australian Department of Health telehealth project. J Telemed Telecare 2005;11(Suppl 2):S19-21.

20. Bashshur RL, Reardon TG, Shannon GW. Telemedicine: A New Health Care Delivery System. Annu Rev Public Health 2000;21:613-37.

21. Hailey D, Ohinmaa A, Roine R. Study quality and evidence of benefit in recent assessments of telemedicine. J Telemed Telecare 2004; 10:318-24.

22. Chipps J, Brysiewicz P, Mars M. A systematic review of the effectiveness of videoconference based tele-education for medical and nursing education. Worldviews Evid Based Nurs 2012;2:78-87.

23. Yozwiak JA, Robiner WN, Victor AM, et al. Videoconferencing at Psychology Internships: Interns' perceptions of interactive television experiences and prospects. J Clin Psychol Med Settings 2010;17:238-48.

24. Moffatt JJ, Eley DS. The reported benefits of telehealth for rural Australians. Aust Health Rev 2010;34:276-81.

25. Wearne SM, Dornan T, Teunissen PW, et al. Supervisor continuity or co-location: which matters in residency education? Findings from a qualitative study of remote supervisor family physicians in Australia and Canada. Acad Med 2014. doi:10.1097/ACM.0000000000000587 [Epub ahead of print 2 Dec 2014].

26. Cameron MP, Ray R, Sabesan S. Physicians' perceptions of clinical supervision and educational support via videoconference: a systematic review. J Telemed Telecare 2014;20:272-81.
27. Moran AM, Coyle J, Pope R, et al. Supervision, support and mentoring interventions for health practitioners in rural and remote contexts: an integrative review and thematic synthesis of the literature to identify mechanisms for successful outcomes. Hum Resour Health 2014;12:10.

28. Sabesan S, Simcox K, Marr I. Medical oncology clinics through videoconferencing: an acceptable telehealth model for rural patients and health workers. Intern Med J 2012;42:780-5.

29. King N. Using templates in the thematic analysis of text. In: Cassell $C$ Symon G, eds. Essential guide to qualitative methods in organizational research. Thousans Oaks: SAGE Publications, 2004:256-70.

30. Crabtree BF, Miller WL. Doing qualitative research. Newbury Park, Calif: Sage Publications, 1992

31. Reeves S, Albert M, Kuper A, et al. Qualitative research: why use theories in qualitative research? BMJ 2008;337:631-4.

32. Smith JA. Semi-structured interviewing and qualitative analysis. In: Smith JA, Harré R, Langenhove LV, eds. Rethinking methods in psychology. London: Sage Publications, 1995:9-26.

33. NVivo for Mac [program]. 2 version. Doncaster, Australia: QSR International Pty Ltd, 2014.

34. Seale C. The quality of qualitative research. London: SAGE, 1999.

35. Kitto S, Chesters J, Grbich C. Quality in qualitative research. Med J Aust 2008;188:243.

36. Barbour RS. Checklists for improving rigour in qualitative research: a case of the tail wagging the dog? BMJ 2001;322:1115-17.

37. Gammon D, Sorlie T, Bergvik S, et al. Psychotherapy supervision conducted via videoconferencing: a qualitative study of users' experiences. J Telemed Telecare 1998:4:33-5.

38. Sargeant J, Allen M, Langille D. Physician perceptions of the effect of telemedicine on rural retention and recruitment. $J$ Telemed Telecare 2004;10:89-93.

39. Wilkinson TJ, Smith JD, Margolis SA, et al. Structured assessment using multiple patient scenarios by videoconference in rural settings. Med Educ 2008;42:480-7.

40. Klein D, Davis P, Hickey L. Videoconferences for rural physicians' continuing health education. J Telemed Telecare 2005;11(Suppl 1): 97-9.

41. Finley JP, Beland MJ, Boutin C, et al. A national network for the tele-education of Canadian residents in pediatric cardiology. Cardiol Young 2001;11:526-31.

42. Wood JAV, Miller TW, Hargrove DS. Clinical supervision in rural settings: a telehealth model. Prof Psychol Res Pract 2005;36:173-9.

43. Wearne SM, Teunissen PW, Dornan T, et al. Physical isolation with virtual support: Registrars' learning via remote supervision. Med Teach 2014:1-7. doi:10.3109/0142159X.2014.947941 [Epub ahead of print 26 Aug 2014]

44. Callas PW, Ricci MA, Caputo MP. Improved rural provider access to continuing medical education through interactive videoconferencing. Telemed J E Health 2000;6:393-9.

45. Gagnon MP, Duplantie J, Fortin JP, et al. Implementing telehealth to support medical practice in rural/remote regions: what are the conditions for success? Implement Sci 2006;1:18.

46. Ray RA, Fried O, Lindsay D. Palliative care professional education via video conference builds confidence to deliver palliative care in rural and remote locations. BMC Health Serv Res 2014;14:272.

47. Allen M, Sargeant J, Mann K, et al. Videoconferencing for practice-based small-group continuing medical education: feasibility, acceptability, eff 'ectiveness, and cost. J Contin Educ Health Prof 2003;23:38-47.

48. Jennett PA, Affleck Hall L, Hailey D, et al. The socio-economic impact of telehealth: a systematic review. $J$ Telemed Telecare 2003:9:311-20.

49. Robinson A. Video-conferencing: under-used by rural general practitioners. Aust Health Rev 2002;25:131-5.

50. Mooi JK, Whop LJ, Valery PC, et al. Teleoncology for Indigenous patients: the responses of patients and health workers. Aust $J$ Rural Health 2012;20:265-9. 